九州大学学術情報リポジトリ

Kyushu University Institutional Repository

\title{
Records of Some Subcortical Coleoptera New to the Fauna of Korea (Insecta)
}

Chujo, Michitaka

Lee, Chang Eon

https://doi.org/10.5109/2569

出版情報: ESAKIA. 33，pp.123-124，1993-03-31. Entomological Laboratory，Faculty of Agriculture, Kyushu University バージョン :

権利関係 : 


\title{
Records of Some Subcortical Coleoptera New to the Fauna of Korea (Insecta) 1,2)
}

\author{
Michitaka CHỔô \\ Hikosan Biological Laboratory, Faculty of Agriculture, Kyushu \\ University, Hikosan, Japan 824-07 \\ and \\ Chang Eon LeE \\ Department of Biology, College of Natural Sciences, Kyungpook \\ National University, Taegu, 702-701 Korea
}

Four species of Coleoptera, Rhysodes comes Reitter, Prostomis latoris Reitter, Psammoecus triguttatus Reitter and Uleiodes arborea (Reitter) are newly added to the Korean insect fauna.

\section{RHYSODIDAE}

Rhysodes comes (Lewis)

Epiglymmius comes Lewis, 1888, Ann. Mag. Nat. Hist., [6],2(7): 79 (Japan: Honshu \& Hokkaido).

Spec. exam.: 1 ex., Kwangnung, Pochon-gun, Kyonggi-do, Korea, 14-19. v. 1992, M. T. Chûjô leg.

Gen. distr.: Korea \& Japan (Hokkaido, Honshu, Shikoku \& Kyushu)

\section{PROSTOMIDAE}

\section{Prostomis latoris Reitter}

Prostomis latoris Reitter, 1889, Wiener Ent. Zeit., 8(10): 315 (Japan: Honshu).

Spec. exam.: 2 exs., Yongsil, Mt. Hallasan, Chejudo, Korea, 20. v. 1990, M. T. Chûjô leg.

Gen. distr.: Korea (Chejudo Island) \& Japan (Hokkaido, Honshu, Shikoku, Kyushu \& Tsushima Is.).

\section{SILVANIDAE}

1) Results from the Korea-Japan Co-operative Science Program on "The Evolution and Biogeography of the Insects in the East Asia". No. 18.

2) Contribution from the Hikosan Biological Laboratory, Faculty of Agriculture, Kyushu University, Hikosan (Ser. 4, No. 10). 
Psammoecus triguttatus Reitter

Psammoecus triguttatus Reitter, 1874, Verh. Zool.-Bot. Ver. Wien, 24: 524 (Japan).

Spec. exam.: 1 ex.(light trap), Kaewol Bridge, Chejudo, Korea, 23. vii. 1990, M. T. Chûjô leg.

Gen. distr.: Korea (Chejudo Island) \& Japan (Hokkaido, Honshu, Shikoku, Kyushu \& Tsushima Is.).

\section{CUCUJIDAE}

\section{Vleiota arborea (Reitter)}

Hyliota arboreus Reitter, 1889, Wiener Ent. Zeit., 8(10): 317 (Japan: Honshu).

Spec. exam.: 16 exs., Jeonglyong Chi, Sannae Myeon, Namweon Gun, Jeongra-buk Do, Korea, 29. ix. 1991, M. T. Chûjô leg.; 4 exs., Samjeong Ri, Macheong Myeon, Hamyang Gun, Gyeongsang-nam Do, Korea, 29. ix. 1991, M. T. Chûjô leg.

Gen. distr.: Korea \& Japan (Hokkaido, Honshu, Shikoku, Kyushu \& Yakushima Is.).

\section{SERECTED REFERENCE}

Krivolutskaya, G. O., 1992, Cucujidae, In Key to the identification of insects of the Russian Far East, vol. 3, Coleoptera, part 2: 233-245. (in Russian)

Lewis, G., 1888. On the Family Rhysodidae. Ann. Mag. Nat. Hist.,[6],2(7): 79.

Reitter, E., 1874. Beschreibungen neuer Käfer-Arten nebst synonymischen Notizen. Verh.Zool.Bot. Vet: Wien, 24: 524.

1889. Verzeichnis der Cucujiden Japans mit Beschreibungen neuer Arten. Wiener Fnt.Zeit., 8(10): 313-320.

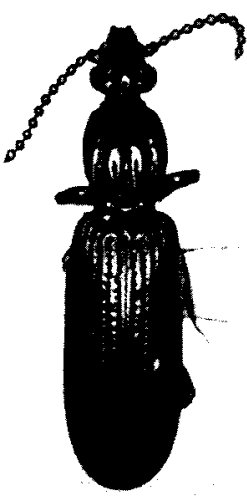

1

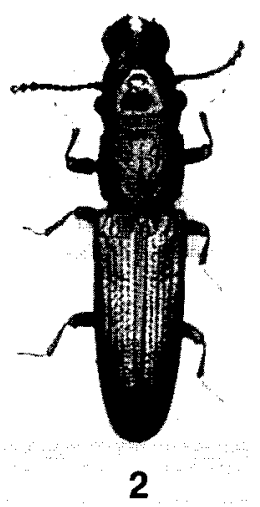

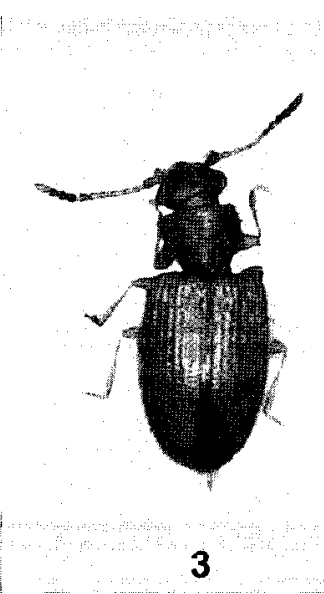

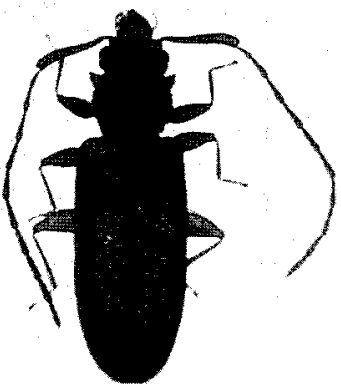

4

Figs. 1: Rysodes comes (Lewis), 2: Prostomis latoris Reitter, 3: Psammoecus triguttatus Reitter, 4: Vleiota arborea (Reitter). 